\title{
Analytical Solutions of Fuzzy Linear Differential Equations in the Conformable Setting
}

\author{
Awais Younus ${ }^{a}$, Muhammad Asif $^{a}$, Usama Atta $^{a}$, Tehmina Bashir $^{a}$, Thabet $^{\circ}$ \\ ABDELJAWAD ${ }^{\mathrm{b}, *}$ (1) \\ a Centre for Advanced Studies in Pure and Applied Mathematics, Bahauddin Zakaryia \\ University, Multan, Pakistan \\ $\mathrm{b}$ Department of Mathematics and General Sciences, Prince Sultan University, 11586 \\ Riyadh,Saudi Arabia
}

- Received: 30 August 2021 • Accepted: 12 December 2021 • Published Online: 30 December 2021

\begin{abstract}
In this paper, we provide the generalization of two predefined concepts under the name fuzzy conformable differential equations. We solve the fuzzy conformable ordinary differential equations under the strongly generalized conformable derivative. For the order $\Psi$, we use two methods. The first technique is to resolve a fuzzy conformable differential equation into two systems of differential equations according to the two types of derivatives. The second method solves fuzzy conformable differential equations of order $\Psi$ by a variation of the constant formula. Moreover, we generalize our results to solve fuzzy conformable ordinary differential equations of a higher order. Further, we provide some examples in each section for the sake of demonstration of our results.
\end{abstract}

Keywords: Fuzzy-valued function, Initial value problem, Strongly generalized conformable derivative. MSC: 34A08, 34A07, 34K36.

\section{Introduction}

Any or most of the data may sometimes be ambiguous when modeling real-world phenomena. The initial state may not be known, for instance, or knowledge about different parameters needed as part of the model may be uncertain. A natural way of modeling is fuzzy differential equations in this case.

The term "fuzzy differential equation" was coined in 1987 by Kandel and Byatt [1]. To study the solution of fuzzy initial and boundary value problems, we need the concept of derivative of fuzzy-valued functions [2]. The first attempt, in this case, was H-derivative, which had its starting point in the Hukuhara derivative of multi-valued functions and then extended for fuzzy-valued functions [3]. Using Hukuhara derivative, [4] and [5] 
started to develop the theory of fuzzy differential equations. But this technique had the disadvantage that it does not exist for all fuzzy-valued functions. Therefore, Bede [6] introduced a generalization of $\mathrm{H}$-derivative based on the lateral type of derivatives called strongly generalized differentiability. This concept allows us to cope with the above-mentioned shortcomings. So we use this differentiability concept in the present paper.

Modeling with fuzzy differential equations can help us to understand our problem in a better way. Several authors have solved real-life problems with fuzzy differential equations. See for example, ([7], [8], [9], [10]). There are almost 25 definitions of the fractional derivative. The most famous of them are Caputo and R-L derivatives. Both these definitions include integral in their definitions. These definitions inherit only linearity property from the usual derivative [11]. Therefore, the solution of differential equations is difficult to obtain using these definitions, see for example [12]. To overcome these disadvantages, Khalil [13] introduced a new definition of fractional derivative called conformable derivative. This new definition differs from other fractional derivatives and is similar to the classical one. Fractional derivatives have no geometrical interpretation because of their non-local behavior but conformable derivative inherits the local behavior of the usual derivative and possesses the geometrical interpretation [14].

Conformal differential equations can best describe certain real-world phenomena ([15], [16], [17], [18]). This is understandable because structures are typically not flawless and can be broken (such as friction, coercion, external forces, etc. and integer-order derivatives may not be appropriate to understand the state variables' trajectories because of this. We have infinite choices of derivative orders to consider by considering fractional derivatives, and with this, we can determine what the fractional differential equation is that we can use to model our phenomenon. For more research works and details on relevant concept, see for example ([19], [20], [21], [22], [23]).

Fuzzy conformable derivative of order $\Psi$ was introduced by ([24], [25]) and used in [26] to solve fuzzy conformable differential equations. But they solved fuzzy differential equations with numerical techniques. In this paper, we have used the strongly generalized conformable derivative to solve fuzzy conformable differential equations. Moreover, we obtain solutions of higher-order fuzzy fractional differential equations of conformable type.

We arrange the rest of this article in the following form: Section 2 includes the basic concepts needed for the rest of the manuscript. In sections 3 to 5, we have solved the fuzzy conformable differential equations with different orders. In the end, we conclude the article with brief comments.

\section{Basic Concepts}

In this section, we will present the basic concepts which we will use in the major part of the article. A fuzzy set is a map $\eta: \mathbb{R} \rightarrow[0,1]$ which generalizes classical sets from $\{0,1\}$ to $[0,1]$. A fuzzy number $\eta$ is a fuzzy set that satisfies some additional properties of convexity, normality, upper-semicontinuity, and compact support. We use $\mathbb{R}_{\Phi}$ to denote the space of all real fuzzy numbers [27]. For $0 \leqslant \gamma<1, \gamma$-cuts for a fuzzy number $\eta$ is defined as $(\eta, \gamma)=\{v \in \mathbb{R}: \eta(v) \geqslant \gamma\}$. In $\gamma$-cuts form, the fuzzy number $\eta$ is 
represented in the form $(\eta, \gamma)=\left[\left(\eta_{*}, \gamma\right),\left(\eta^{*}, \gamma\right)\right]$. A triangular fuzzy number $\eta$, denoted by an ordered triple $(a, b, c)$, with the condition $a \leqslant b \leqslant c$. The $\gamma$-cuts associated with triangular fuzzy number $\eta$ are $[a+(b-a) \gamma, c-(c-b) \gamma]$. The Hausdorff metric $\mathrm{D}_{\mathrm{H}}: \mathbb{R}_{\Phi} \times \mathbb{R}_{\Phi} \rightarrow \mathbb{R}_{0}^{+}$on the space of fuzzy numbers is defined as

$$
D_{H}(\eta, v)=\sup _{0<\gamma \leqslant 1} \max \left\{\left|\left(\eta_{*}, \gamma\right)-\left(v_{*}, \gamma\right)\right|,\left|\left(\eta^{*}, \gamma\right)-\left(v^{*}, \gamma\right)\right|\right\}
$$

If $\eta, v \in \mathbb{R}_{\Phi}$, then addition on the space of fuzzy numbers by $\gamma$-cuts is defined as $(\eta+v, \gamma)=\left[\left(\eta_{*}, \gamma\right)+\left(v_{*}, \gamma\right),\left(\eta^{*}, \gamma\right)+\left(v^{*}, \gamma\right)\right]$. The H-difference for two fuzzy numbers $\eta$ and $v$ denoted by $\eta \ominus v$ and defined as a fuzzy number $\omega$ such that $\omega=$ $\eta+v$. In $\gamma$-cuts form, H-difference for two fuzzy numbers $\eta$ and $v$ has the form $(\eta \ominus v, \gamma)=\left[\left(\eta_{*}, \gamma\right)-\left(v_{*}, \gamma\right),\left(\eta^{*}, \gamma\right)-\left(v^{*}, \gamma\right)\right]$. A fuzzy-valued function $\Phi$ is a function from arbitrary interval to $\mathbb{R}_{\Phi}$. In $\gamma$-cuts form, $\Phi$ is represented in the form $\Phi(v, \gamma)=$ $\left[\Phi_{*}(\nu, \gamma), \Phi^{*}(\nu, \gamma)\right]$

Now, we present the definition of the derivative, which we have to use in our paper to solve the fuzzy conformable differential equation [24].

A fuzzy-valued function $\Phi$ is called strongly generalized conformable differentiable of order $\Psi$ at a point $v \in[0, \infty)$ if

1. $(\forall) \theta>0$, H-difference $\Phi\left(v+\theta v^{1-\Psi}\right) \ominus \Phi(v)$ and $\Phi(v) \odot \Phi\left(v-\theta v^{1-\Psi}\right)$ exist and we have

$$
\lim _{\theta \rightarrow 0} \frac{\Phi\left(v+\theta v^{1-\Psi}\right) \ominus \Phi(v)}{\theta}=\lim _{\theta \rightarrow 0} \frac{\Phi(v) \ominus \Phi\left(v-\theta v^{1-\Psi}\right)}{\theta} .
$$

2. $(\forall) \theta>0$, H-differences $\Phi(v) \odot \Phi\left(\nu+\theta v^{1-\Psi}\right)$ and $\Phi\left(\nu-\theta v^{1-\Psi}\right) \ominus \Phi(v)$ exist and we have

$$
\lim _{\theta \rightarrow 0} \frac{\Phi(v) \ominus \Phi\left(v+\theta v^{1-\Psi}\right)}{\theta}=\lim _{\theta \rightarrow 0} \frac{\Phi\left(v-\theta v^{1-\Psi}\right) \ominus \Phi(v)}{\theta} .
$$

We say that a fuzzy-valued function $\Phi$ is differentiable of type

1. ( $\Psi-1)$, if $\Phi$ is differentiable in the first case.

2. ( $\Psi-2)$, if $\Phi$ is differentiable in the second case.

A fuzzy-valued function $\Phi$ is continuous if $(\forall) \theta>0$, there exists $v>0$ such that

$$
\left|\Phi(v)-\Phi\left(v_{0}\right)\right|<\theta \text { whenever }\left|v-v_{0}\right|<v
$$

Mathematically, we express it as

$$
\lim _{v \rightarrow v_{0}} \Phi(v)=\Phi\left(v_{0}\right)
$$

For a fuzzy-valued function $\Phi$, the fuzzy conformable integral of order $\Psi$ is defined as

$$
\mathrm{I}^{\Psi} \Phi(v)=\int_{0}^{v} \Phi(\mu) \mu^{\Psi-1} \mathrm{~d} \mu,
$$

where integration is in fuzzy Riemann sense. 
For a fuzzy-valued function $\Phi$ with the $\gamma$-cuts form

$$
\Phi(v, \gamma)=\left[\Phi_{*}(\nu, \gamma), \Phi^{*}(\nu, \gamma)\right]
$$

If $\Phi_{*}(v)$, and $\Phi^{*}(v)$ are conformable Riemann integrable, then $\Phi$ itself is fuzzy conformable Riemann integrable such that its integral is a fuzzy number and its integral is the integral of $\Phi_{*}$ and $\Phi^{*}$ expressed in the form

$$
\int_{0}^{v} \Phi(v) v^{\Psi-1} \mathrm{~d} v=\left[\int_{0}^{v} \Phi_{*}(v) v^{\Psi-1} \mathrm{~d} v, \int_{0}^{v} \Phi^{*}(v) v^{\Psi-1} \mathrm{~d} v\right]
$$

Theorem 2.1. (First Fundamental Theorem) For a fuzzy conformable integrable fuzzy-valued function $\Phi$, we have

1. If $\Phi$ is differentiable of the type ( $\Psi-1)$, then

$$
\int_{0}^{v} \Phi^{\Psi}(v) v^{\Psi-1} \mathrm{~d} v=\Phi(v)
$$

2. If $\Phi$ is differentiable of the type ( $\Psi-2)$, then

$$
\int_{0}^{v} \Phi^{\Psi}(v) v^{\Psi-1} \mathrm{~d} v \odot \Phi(v)=0 .
$$

Proof. We provide proof for the first case. The second case can be proved in a similar pattern.

The relationship between the strongly generalized conformable derivative and conformable derivative allows us to have the following

$$
\int_{0}^{\nu} \Phi^{\Psi}(\mu, \gamma) \mu^{\Psi-1} \mathrm{~d} \mu=v^{1-\Psi} \int_{0}^{\nu} \Phi^{\prime}(\mu, \gamma) \mu^{\Psi-1} \mathrm{~d} \mu .
$$

Using the relationship between $\Phi$ and $\Phi_{*}, \Phi^{*}$, we have

$$
\int_{0}^{v} \Phi^{\Psi}(\mu, \gamma) \mu^{\Psi-1} \mathrm{~d} \mu=v^{1-\Psi}\left[\int_{0}^{v}\left(\Phi_{*}\right)^{\prime}(\mu, \gamma) \mu^{\Psi-1} \mathrm{~d} \mu, \int_{0}^{v}\left(\Phi^{*}\right)^{\prime}(\mu, \gamma) \mu^{\Psi-1} \mathrm{~d} \mu\right] .
$$

Since derivative and integration are the inverse processes of each other, so cancel each other.

$$
\int_{0}^{v} \Phi^{\Psi}(\mu, \gamma) \mu^{\Psi-1} \mathrm{~d} \mu=v^{1-\Psi} v^{\Psi-1} \Phi_{*}(\nu, \gamma), v^{1-\Psi} v^{\Psi-1} \Phi^{*}(\nu, \gamma) .
$$

So we have our required result in the form

$$
\int_{0}^{v} \Phi^{\Psi}(\mu, \gamma) \mu^{\Psi-1} \mathrm{~d} \mu=\Phi(\nu, \gamma)
$$

Theorem 2.2. (Second Fundamental Theorem) For a fuzzy conformable integrable fuzzy-valued function $\Phi$, we have 
1. If $\Phi$ is differentiable of the type $(\Psi-1)$, then

$$
\int_{a}^{v} \Phi^{\Psi}(\mu) \mu^{\Psi-1} \mathrm{~d} \mu=\Phi(v) \ominus \Phi(a) .
$$

2. If $\Phi$ is differentiable of the type ( $\Psi-2)$, then

$$
\Phi(v)=\Phi(a) \ominus \int_{a}^{v} \Phi^{\Psi}(\mu) \mu^{\Psi-1} \mathrm{~d} \mu .
$$

Proof. For proof, see [24].

Now we generalize the second fundamental theorem as follows:

Theorem 2.3. For a continuous fuzzy-valued function $\Phi^{\mathrm{p} \Psi}$, we have

1. Consider $\Phi^{\mathrm{p} \Psi}, \mathrm{p}=1,2, \cdots, \mathrm{k}$ are strongly generalized conformable differentiable of the type ( $\Psi-1)$, and the type of derivative same, then we have

$$
\Phi_{(\Psi-1)}^{(p-1) \Psi}(b)=\Phi_{(\Psi-1)}^{(p-1) \Psi}(a)+\int_{a}^{b} \Phi_{(\Psi-1)}^{p \Psi}(v) v^{\Psi-1} d v
$$

2. If $\Phi^{p^{\Psi}}, p=1,2, \cdots, k$ are strongly generalized conformable differentiable of the type $(\Psi-2)$, and the type of derivative same, then we have

$$
\Phi_{(\Psi-2)}^{(p-1) \Psi}(b)=\Phi_{(\Psi-2)}^{(p-1) \Psi}(a)+\int_{a}^{b} \Phi_{(\Psi-2)}^{p \Psi}(v) v^{\Psi-1} d v
$$

Proof. We prove the case (2) here. Case (1) can be proved similarly.

Since a continuous fuzzy-valued function is fuzzy conformable integrable, so we have

$$
\int_{a}^{b} \Phi_{(\Psi-2)}^{p \Psi}(v) v^{\Psi-1} \mathrm{~d} v=\left[\int_{a}^{b}\left(\Phi^{*}\right)^{p \Psi}(v) v^{\Psi-1} \mathrm{~d} v, \int_{a}^{b}\left(\Phi_{*}\right)^{p \Psi}(v) v^{\Psi-1} \mathrm{~d} v\right]
$$

Using Theorem 2.2, we have

$$
\begin{gathered}
\int_{a}^{b} \Phi_{(\Psi-2)}^{p \Psi}(v) v^{\Psi-1} d v=\left[\left(\Phi^{*}\right)^{(p-1) \Psi}(b)-\left(\Phi^{*}\right)^{(p-1) \Psi}(a),\right. \\
\left.\left(\Phi_{*}\right)^{(p-1) \Psi}(b)-\left(\Phi_{*}\right)^{(p-1) \Psi}(a)\right] .
\end{gathered}
$$

We can write as

$$
\begin{gathered}
\int_{a}^{b} \Phi_{(\Psi-2)}^{p \Psi}(v) v^{\Psi-1} \mathrm{~d} v=\left[\left(\Phi^{*}\right)^{(p-1) \Psi}(b),\left(\Phi_{*}\right)^{(p-1) \Psi}(b)\right] \Theta \\
{\left[\left(\Phi^{*}\right)^{(p-1) \Psi}(a),\left(\Phi_{*}\right)^{(p-1) \Psi}(a)\right] .}
\end{gathered}
$$

Thus we have

$$
\Phi_{(\Psi-2)}^{(p-1) \Psi}(b)=\Phi_{(\Psi-2)}^{(p-1) \Psi}(a)+\int_{a}^{b} \Phi_{(\Psi-2)}^{p \Psi}(v) v^{\Psi-1} d \nu
$$


Theorem 2.4. Let $\Phi$ be a continuous fuzzy-valued function. Define a fuzzy-valued function $v(v)$ in the form

$$
v(v)=\eta \odot \int_{0}^{\nu}-\left(\Phi(\mu) \mu^{\Psi-1} \mathrm{~d} \mu\right),
$$

then $v(v)$ is differentiable of type ( $\Psi-2)$ and its strongly generalized conformable generalized derivative is

$$
v^{\Psi}(v)=\Phi(v)
$$

\section{Fuzzy Conformable ODEs of Order $\Psi$}

Now, we present the solution of fuzzy conformable solutions using the strongly generalized conformable derivative. We divide this section into two parts. In the first part, we show that the solution of the fuzzy conformable differential equation of order $\Psi$ and the associated systems of differential equations are equivalent. In the second part, we solve the fuzzy conformable differential equation of order $\Psi$ with the variation of the constant formula.

Consider the fuzzy conformable initial value problem of order $\Psi$ in the form

$$
\begin{aligned}
& \digamma^{\Psi}(v)=\Phi(v, \digamma(v)), \\
& \digamma(0)=\eta,
\end{aligned}
$$

where $\Phi$ is a continuous fuzzy-valued function and $\eta$ is a fuzzy number.

Solution of the equation (3.1) can be determined in two possible ways:

Case 1: If $\digamma(v)$ is differentiable of type $(\Psi-1)$, then we have

$$
\digamma^{\Psi}(\nu, \gamma)=\left[\left(\digamma_{*}\right)^{\Psi}(\nu, \gamma),\left(\digamma^{*}\right)^{\Psi}(\nu, \gamma)\right] .
$$

So the equation (3.1) can be translated into the following system of conformable differential equations:

$$
\begin{aligned}
& \left(\digamma_{*}\right)^{\Psi}(\nu, \gamma)=\Phi_{*}(\nu, \gamma), \\
& \left(\digamma^{*}\right)^{\Psi}(\nu, \gamma)=\Phi^{*}(\nu, \gamma), \\
& \left(\digamma^{*}\right)(0, \gamma)=\left(\eta_{*}, \gamma\right), \\
& \left(\digamma^{*}\right)(0, \gamma)=\left(\eta^{*}, \gamma\right) .
\end{aligned}
$$

Case 2: If $\digamma(v)$ is differentiable of type ( $\Psi-2)$, then

$$
\digamma^{\Psi}(\nu, \gamma)=\left(\digamma^{*}\right)^{\Psi}(\nu, \gamma),\left(\digamma_{*}\right)^{\Psi}(\nu, \gamma) \text {. }
$$

So equation (3.1) can be translated into the following system of conformable differential equations:

$$
\begin{aligned}
& \left(\digamma_{*}\right)^{\Psi}(v, \gamma)=\Phi^{*}(v, \gamma), \\
& \left(\digamma^{*}\right)^{\Psi}(v, \gamma)=\Phi_{*}(\nu, \gamma), \\
& \left(\digamma_{*}\right)(0, \gamma)=\left(\eta_{*}, \gamma\right), \\
& \left(\digamma^{*}\right)(0, \gamma)=\left(\eta^{*}, \gamma\right) .
\end{aligned}
$$

If the solution obtained from the case (1) and case (2) is a valid fuzzy number, then we obtain the solution of the equation (3.1) in the form $\digamma(v, \gamma)=\left[\digamma_{*}(v, \gamma), \digamma^{*}(v, \gamma)\right]$. 
Definition 3.1. Consider a fuzzy-valued function $\digamma$ such that both types of derivative exist for $\digamma$, then

1. If $\digamma$ and its strongly generalized conformable derivative of type ( $\Psi-1)$ satisfy equation (3.1), then $\digamma$ is called the solution of type ( $\Psi-1)$.

2. If $\digamma$ and its strongly generalized conformable derivative of type ( $\Psi-2)$ satisfy equation (3.1), then $\digamma$ is called the solution of type ( $\Psi-2)$.

Example 3.2. Consider a fuzzy conformable initial value problem of order $\Psi$

$$
\begin{aligned}
& \Phi^{\Psi}(\nu)=-\Phi(v)+1+v, \\
& \Phi(0, \gamma)=[1+\gamma, 3-\gamma] .
\end{aligned}
$$

Case 1: If $\Phi$ is differential of type ( $\Psi-1)$, then the equation (3.4) can be written in the following system of conformable differential equations of order $\Psi$

$$
\begin{aligned}
& \left(\Phi_{*}\right)^{\Psi}(v, \gamma)=-\Phi^{*}(v, \gamma)+1+v, \\
& \left(\Phi^{*}\right)^{\Psi}(\nu, \gamma)=-\Phi_{*}(v, \gamma)+1+v .
\end{aligned}
$$

Solving the above system of the conformable differential equations, we obtain the solution in the form

$$
\begin{gathered}
\Phi_{*}(\nu, \gamma)=-\sinh \frac{\nu^{\Psi}}{\Psi}(3-\gamma)-\cosh \frac{\nu^{\Psi}}{\Psi}(1+\gamma)+\frac{\nu^{\Psi}}{\Psi}+i \sin i \frac{\nu^{\Psi}}{\Psi}-\sinh \frac{\nu^{\Psi}}{\Psi} \\
\Phi^{*}(\nu, \gamma)=\cosh \frac{\nu^{\Psi}}{\Psi}(3-\gamma)-\sinh \frac{\nu^{\Psi}}{\Psi}(1+\gamma)+\frac{\nu^{\Psi}}{\Psi}+i \sin i \frac{\nu^{\Psi}}{\Psi}+\sinh \frac{\nu^{\Psi}}{\Psi}
\end{gathered}
$$

Case 2: If $\Phi$ is differential of type ( $\Psi-2)$, then the equation (3.4) can be written in the following system of conformable differential equations of order $\Psi$

$$
\begin{aligned}
& \left(\Phi_{*}\right)^{\Psi}(v, \gamma)=-\Phi^{*}(v, \gamma)+1+v, \\
& \left(\Phi^{*}\right)^{\Psi}(\nu, \gamma)=-\Phi_{*}(v, \gamma)+1+v .
\end{aligned}
$$

Solving the above system of the conformable differential equations, we obtain the solution in the form

$$
\begin{aligned}
& \Phi_{*}(\nu, \gamma)=(1+\gamma) e^{-\frac{v^{\Psi}}{\Psi}}+\frac{v^{\Psi}}{\Psi}, \\
& \Phi^{*}(\nu, \gamma)=(3-\gamma) e^{-\frac{v^{\Psi}}{\Psi}}+\frac{\nu^{\Psi}}{\Psi} .
\end{aligned}
$$

Lemma 3.3. ([24]) The fuzzy conformable differential equation (3.1) is equivalent to one of the following fuzzy conformable integral equations according to the type of $(\Psi-1)$ or ( $\Psi-2)$ differentiability.

1. $\digamma(v)=\eta+\int_{0}^{v} \Phi(\mathrm{q}) \mathrm{q}^{\Psi-1} \mathrm{dq}$.

2. $\digamma(v)=\eta \odot(-1) \int_{0}^{v} \Phi(\mathrm{q}) \mathrm{q}^{\mathrm{w}-1} \mathrm{dq}$.

Theorem 3.4. ([24]) Consider fuzzy conformable differentiable equation (3.1) with $\gamma$-cuts in the form

$$
\Phi(v, \gamma)=\left[\Phi_{*}(v, \gamma), \Phi^{*}(v, \gamma)\right]
$$

if $\Phi_{*}$ and $\Phi^{*}$ are equi-continuous and satisfy the Lipschitz condition, then the solution of equation (3.1) and both associated systems of conformable differential equations are equivalent. 


\subsection{Variation of Constant Formula}

Now, we use the variation of the constant formula to solve the fuzzy conformable differential equations. For this, we take the fuzzy conformable initial value problem of order $\Psi$ in the form

$$
\begin{aligned}
& \Phi^{\Psi}(v)=\alpha(v) \odot \Phi(v)+\beta(v), \\
& \Phi(0)=v,
\end{aligned}
$$

where $\alpha \in \mathbb{R}, v$ is a fuzzy number and $\beta$ is a continuous fuzzy-valued function.

Definition 3.5. Consider a fuzzy-valued function $\Phi$, such that both types of derivatives exist for $\Phi$, then

1. If $\Phi$ and its derivative of type ( $\Psi-1)$ satisfy the equation (3.5), then $\Phi$ is called the solution of type $(\Psi-1)$.

2. If $\Phi$ and its derivative of type ( $\Psi-2)$ satisfy the equation (3.5), then $\Phi$ is called the solution of type ( $\Psi-2)$.

Now to solve the equation (3.5), we have three possibilities. We solve our problem with $\alpha>0, \alpha<0$, and $\alpha=0$.

Case 1: For $\alpha<0$, we have two cases.

(i) When $\Phi$ is differentiable of type ( $\Psi-1)$, then the equation (3.5) can be converted into the following system of conformable differential equations

$$
\begin{aligned}
& \left(\Phi_{*}\right)^{\Psi}(v)=\alpha(v) \cdot \Phi^{*}(v)+\beta_{*}(v), \\
& \left(\Phi^{*}\right)^{\Psi}(v)=\alpha(v) \cdot \Phi_{*}(v)+\beta^{*}(v), \\
& \Phi_{*}\left(v_{0}\right)=v_{*}, \\
& \Phi^{*}\left(v_{0}\right)=v^{*} .
\end{aligned}
$$

We can write the above system of conformable ordinary differential equations in the form

$$
\left[\begin{array}{l}
\left(\Phi_{*}\right)^{\Psi}(v) \\
\left(\Phi^{*}\right)^{\Psi}(v)
\end{array}\right]=\left[\begin{array}{cc}
0 & \alpha(v) \\
\alpha(v) & 0
\end{array}\right]\left[\begin{array}{l}
\Phi_{*}(v) \\
\Phi^{*}(v)
\end{array}\right]+\left[\begin{array}{l}
\beta_{*}(v) \\
\beta^{*}(v)
\end{array}\right]
$$

So the above system obtains the form

$$
\Phi^{\Psi}(v)=\mathrm{C}(v) \Phi(v)+\mathrm{D}(v)
$$

where $\Phi(v)=\left(\left[\begin{array}{l}\Phi_{*}(v) \\ \Phi^{*}(v)\end{array}\right]\right), C(v)=\left[\begin{array}{cc}0 & \alpha(v) \\ \alpha(v) & 0\end{array}\right]$ and D $(v)=\left[\begin{array}{l}\beta_{*}(v) \\ \beta^{*}(v)\end{array}\right]$.

Using the variation of constant formula for conformable ordinary differential equations, we obtain the expression in the form

$$
\Phi(v)=e^{\int_{0}^{\nu} C(\mu) \mu^{\Psi-1} d \mu}\left(v+e^{-\int_{0}^{\nu} C(\mu) \mu^{\Psi-1} d \mu} D(r) r^{\Psi-1} d r\right) .
$$


Conformable Maclaurin's of $\sinh v$ and $\cosh v$ helps us to obtain the form

$$
\begin{aligned}
& \left(\begin{array}{l}
\Phi_{*}(v) \\
\Phi^{*}(v)
\end{array}\right)=\left\{\left[\begin{array}{ll}
\cosh \left(\int_{0}^{\nu} \alpha(\mu) \mu^{\Psi-1} \mathrm{~d} \mu\right) & \sinh \left(\int_{0}^{\nu} \alpha(\mu) \mu^{\Psi-1} \mathrm{~d} \mu\right) \\
\sinh \left(\int_{0}^{\nu} \alpha(\mu) \mu^{\Psi-1} \mathrm{~d} \mu\right) & \cosh \left(\int_{0}^{\nu} \alpha(\mu) \mu^{\Psi-1} \mathrm{~d} \mu\right)
\end{array}\right] \times\left[\begin{array}{l}
\Phi_{*} \\
\Phi^{*}
\end{array}\right]+\right. \\
& \left.\int_{0}^{v}\left[\begin{array}{cc}
\cosh \left(\int_{0}^{r} \alpha(\mu) \mu^{\Psi-1} \mathrm{~d} \mu\right) & -\sinh \left(\int_{0}^{r} \alpha(\mu) \mu^{\Psi-1} \mathrm{~d} \mu\right) \\
-\sinh \left(\int_{0}^{r} \alpha(\mu) \mu^{\Psi-1} \mathrm{~d} \mu\right) & \cosh \left(\int_{0}^{r} \alpha(\mu) \mu^{\Psi-1} \mathrm{~d} \mu\right)
\end{array}\right]\left[\begin{array}{l}
\beta_{*}(r) \\
\beta^{*}(\mathrm{r})
\end{array}\right] \mathrm{r}^{\Psi-1} \mathrm{dr}\right\} .
\end{aligned}
$$

Thus for $\alpha<0$, we obtain the solution of equation (3.5) in the form

$$
\begin{gathered}
\Phi(v)=\cosh \left(\int_{0}^{v} \alpha(\mu) \mu^{\Psi-1} \mathrm{~d} \mu\right) \\
\left(v+\int_{0}^{v}\left[\beta(r) \cosh \left(\int_{0}^{r} \alpha(\mu) \mu^{\Psi-1} \mathrm{~d} \mu\right) \ominus \beta(r) \sinh \left(\int_{0}^{r} \alpha(\mu) \mu^{\Psi-1} \mathrm{~d} \mu\right)\right] r^{\Psi-1} \mathrm{dr}\right) \\
+\sinh \left(\int_{0}^{v} \alpha(\mu) \mu^{\Psi-1} \mathrm{~d} \mu\right) \\
\left(v+\int_{0}^{v}\left[\beta(r) \cosh \left(\int_{0}^{r} \alpha(\mu) \mu^{\Psi-1} \mathrm{~d} \mu\right) \odot \beta(r) \sinh \left(\int_{0}^{r} \alpha(\mu) \mu^{\Psi-1} \mathrm{~d} \mu\right)\right] \mathrm{r}^{\Psi-1} \mathrm{dr}\right),
\end{gathered}
$$

with the condition that H-difference

$$
\beta(r) \cosh \left(\int_{0}^{r} \alpha(\mu) \mu^{\Psi-1} d \mu\right) \ominus \beta(r) \sinh \left(\int_{0}^{r} \alpha(\mu) \mu^{\Psi-1} d \mu\right) r^{\Psi-1} d r
$$

must exist. Then this solution is differentiable of type ( $\Psi-1)$.

(ii) If $\Phi$ is differentiable of type ( $\Psi-2)$, then the solution of type ( $\Psi-2)$ has the form

$$
\Phi(v)=e^{\int_{0}^{v} \alpha(\mu) \mu^{\Psi-1} \mathrm{~d} \mu}\left(v \odot \int_{0}^{v}(-\beta(r)) \cdot e^{-\int_{0}^{r} \alpha(\mu) \mu^{\Psi-1} \mathrm{~d} \mu r^{\Psi-1} \mathrm{~d} r}\right),
$$

with the condition that H-difference must exist.

Proof. Since $\left(v+\int_{0}^{v} \beta(\mu) \cdot e^{-\int_{0}^{\mu} \alpha(r) r^{\Psi-1} d r} \mu^{\Psi-1} d \mu\right)$ is differentiable of type ( $\left.\Psi-1\right)$ being a constant function, therefore using Theorem 2.4, its derivative is

$$
\left(v+\int_{0}^{v} \beta(\mu) \cdot e^{-\int_{0}^{\mu} \alpha(r) r^{\Psi-1} d r} \mu^{\Psi-1} d \mu\right)^{\Psi}=\beta(v) \cdot e^{-\int_{0}^{v} \alpha(r) r^{\Psi-1} d r} .
$$

If H-difference $\left(v \odot \int_{0}^{v}(-\beta(\mu)) \cdot e^{-\int_{0}^{\mu} \alpha(r) r^{\Psi-1} d r} \mu^{\Psi-1} d \mu\right)$ exists, then it is differentiable of type ( $\Psi-2)$ and by Theorem 2.4 , its derivative is

$$
\left(v \odot \int_{0}^{v}(-\beta(\mu)) \cdot e^{-\int_{0}^{\mu} \alpha(r) r^{\Psi-1} d r} \mu^{\Psi-1} \mathrm{~d} \mu\right)^{\Psi}=\beta(v) \cdot e^{-\int_{0}^{v} \alpha(r) r^{\Psi-1} \mathrm{dr}} .
$$

Now, put $\Phi(v)=e^{v} \alpha(\mu) \mu^{\psi-1} \mathrm{~d} \mu$, and $\psi(v)=v+\int_{0}^{v} \beta(\mu) \cdot e^{-\int_{0}^{\mu} \alpha(r) r^{\psi-1} d r}$. Since $\alpha(v)>$ 0 , we get $\Phi(v) \cdot \Phi^{\Psi}(\nu)>0$, so we have

$$
\Phi^{\Psi}(v)=\alpha(v) \cdot \Phi(v)+\beta(v) .
$$

This proves our result. 
Case 2: Now we take $\alpha>0$. Similar to case (1), we have two cases according to the two types of differentiability.

(i) If $\Phi$ is differentiable of type ( $\Psi-1)$, then the equation (3.5) can be changed into the following system of conformable differential equations

$$
\begin{aligned}
& \left(\Phi_{*}\right)^{\Psi}(v)=\alpha(v) \cdot \Phi_{*}(v)+\beta_{*}(v), \\
& \left(\Phi^{*}\right)^{\Psi}(v)=\alpha(v) \cdot \Phi^{*}(v)+\beta^{*}(v), \\
& \Phi_{*}\left(v_{0}\right)=v_{*}, \\
& \Phi^{*}\left(v_{0}\right)=v^{*} .
\end{aligned}
$$

Solving the above system of conformable differential equations, we obtain the solution of type $(\Psi-1)$ in the form

$$
\Phi(v)=e^{\int_{0}^{v} \alpha(\mu) \mu^{\Psi-1} \mathrm{~d} \mu}\left(v+\int_{0}^{v} \beta(r) \cdot e^{-\int_{0}^{r} \alpha(\mu) \mu^{\psi-1} \mathrm{~d} \mu} \mathrm{r}^{\Psi-1} \mathrm{~d} r\right) .
$$

(ii) If $\Phi$ is differentiable of type $(\Psi-2)$, then we have the system of conformable differential equations in the form

$$
\begin{aligned}
& \left(\Phi_{*}\right)^{\Psi}(v)=\alpha(v) \cdot \Phi^{*}(v)+\beta^{*}(v), \\
& \left(\Phi^{*}\right)^{\Psi}(v)=\alpha(v) \cdot \Phi_{*}(v)+\beta_{*}(v), \\
& \Phi_{*}\left(v_{0}\right)=v_{*}, \\
& \Phi^{*}\left(v_{0}\right)=v^{*} .
\end{aligned}
$$

Solving the above system, we obtain the solution in the form

$$
\begin{gathered}
\Phi(v)=\cosh \left(\int_{0}^{v} \alpha(\mu) \mu^{\Psi-1} \mathrm{~d} \mu\right) \\
\left(v \odot \int_{0}^{v}\left[\beta(r) \cosh \left(\int_{0}^{r} \alpha(\mu) \mu^{\Psi-1} \mathrm{~d} \mu\right)-\beta(r) \sinh \left(\int_{0}^{r} \alpha(\mu) \mu^{\Psi-1} \mathrm{~d} \mu\right)\right] r^{\Psi-1} \mathrm{dr}\right) \\
\ominus-\sinh \left(\int_{0}^{v} \alpha(\mu) \mu^{\Psi-1} \mathrm{~d} \mu\right) \\
\left(v \odot \int_{0}^{v}\left[\beta(r) \cosh \left(\int_{0}^{r} \alpha(\mu) \mu^{\Psi-1} \mathrm{~d} \mu\right)-\beta(r) \sinh \left(\int_{0}^{r} \alpha(\mu) \mu^{\Psi-1} \mathrm{~d} \mu\right)\right] \mathrm{r}^{\Psi-1} \mathrm{dr}\right),
\end{gathered}
$$

with the condition that $\mathrm{H}$-difference

$$
v \odot \int_{0}^{v}\left[\beta(r) \cosh \left(\int_{0}^{r} \alpha(\mu) \mu^{\Psi-1} d \mu\right)-\beta(r) \sinh \left(\int_{0}^{r} \alpha(\mu) \mu^{\Psi-1} d \mu\right)\right] r^{\Psi-1} d r
$$

must exist. Then this solution is differentiable of type ( $\Psi-2)$.

Case 3: If we put $\alpha=0$, then the equation (3.5) becomes

$$
\begin{aligned}
\Phi^{\Psi}(v) & =\beta(v), \\
\Phi(0) & =v .
\end{aligned}
$$


The solution of type ( $\Psi-1)$ obtained has the form

$$
\Phi(v)=v+\int_{0}^{v} \beta(r) r^{\Psi-1} \mathrm{dr} .
$$

If $\mathrm{H}$-difference exists, then the solution of type $(\Psi-2)$ is of the form

$$
\Phi(v)=v \odot \int_{0}^{v}(-\beta(r)) r^{\Psi-1} \mathrm{dr} .
$$

Remark 3.6. Note that solution of type ( $\Psi-1)$ always exists but the solution of type ( $\Psi-2)$ does not. It exists only when $\mathrm{H}$-difference also exists.

Example 3.7. Consider fuzzy conformable initial value problem of order $\Psi$

$$
\begin{aligned}
\Phi^{\Psi}(v) & =\Phi(v), \\
\Phi(0) & =\eta,
\end{aligned}
$$

where $\eta$ is a fuzzy number with $\gamma$-cuts $[\eta]^{\gamma}=[1+\gamma, 3-\gamma]$.

We obtain the solution of type ( $\Psi-1)$ in the form

$$
\Phi(v)=e^{\frac{v^{\Psi}}{\Psi}} \odot \eta,
$$

and the solution of type ( $\Psi-2)$ is

$$
\Phi(v)=\left[\cosh \left(\frac{v^{\Psi}}{\Psi}\right) \odot\left(-\sinh \left(\frac{v^{\Psi}}{\Psi}\right)\right)\right] \odot \eta=e^{-\frac{v^{\Psi}}{\Psi}} \odot \eta .
$$

Example 3.8. Consider fuzzy conformable initial value problem of order $\Psi$

$$
\begin{aligned}
\Phi^{\Psi}(v) & =(-1) \odot \Phi(v)+\eta, \\
\Phi(0) & =(5,7,9) .
\end{aligned}
$$

Comparing the equation (3.8) with equation (3.5), we obtain $\alpha(v)=-1, \beta(v)=\eta$.

Using equation (3.6), we have $\Phi(v)$ in the form

$$
\Phi(v)=e^{-\frac{1}{\psi}\left(\frac{(v)^{\Psi}}{\psi}\right)}\left[(5,7,9) \ominus \int_{0}^{v}\left((-\eta) e^{\frac{1}{\Psi}\left(\frac{(r)^{\Psi}}{\psi}\right)} r^{\Psi-1} \mathrm{dr}\right)\right] .
$$

Taking $\Psi=\frac{1}{2}$ in the above expression, we obtain the solution of type ( $\left.\Psi-2\right)$ in the form

$$
\Phi(v)=e^{-4 \sqrt{v}}\left[(5,7,9) \ominus\left((-\eta) \frac{1}{2} e^{4 \sqrt{v}}-\frac{1}{2}\right)\right] .
$$




\section{Fuzzy Conformable ODEs of Order $2 \Psi$}

The general form of fuzzy conformable initial value problem of order $2 \Psi$ is

$$
\begin{aligned}
& v^{2 \Psi}(v)=\Phi\left(v, v(v), v^{\Psi}(v)\right), \\
& v(0)=\eta_{1}, v^{\Psi}(0)=\eta_{2} .
\end{aligned}
$$

Theorem 4.1. For a continuous fuzzy-valued function $\Phi$, a fuzzy-valued function $v$ is a solution of the equation (4.1) iff there exist continuous fuzzy-valued functions $v$ and $v^{\Psi}$, which satisfy the following equation

1.

$$
v(v)=\int_{0}^{v}\left(\int_{0}^{v} \Phi\left(r, v(r), v^{\Psi}(r)\right) r^{\Psi-1} d r\right) r^{\Psi-1} d r+\eta_{2} \frac{v^{\Psi}}{\Psi}+\eta_{1},
$$

if $v$ and $v^{\Psi}$ are differentiable of type ( $\left.\Psi-1\right)$.

2.

$$
v(v)=\eta_{1} \ominus(-1)\left(\eta_{2} \frac{v^{\Psi}}{\Psi}\right) \ominus(-1) \int_{0}^{v}\left(\int_{0}^{v} \Phi\left(r, v(r), v^{\Psi}(r)\right) r^{\Psi-1} d r\right) r^{\Psi-1} d r,
$$

if $v$ and $v^{\Psi}$ are differentiable of type $(\Psi-2)$.

3.

$$
v(v)=\ominus(-1)\left(\eta_{2} \frac{v^{\Psi}}{\Psi}+\int_{0}^{v}\left(\int_{0}^{v} \Phi\left(r, v(r), v^{\Psi}(r)\right) r^{\Psi-1} d r\right) r^{\Psi-1} d r\right)+\eta_{1}
$$

if $v$ is differentiable of type ( $\Psi-1)$, and $v^{\Psi}$ is differentiable of type ( $\left.\Psi-2\right)$.

4 .

$$
v(v)=\eta_{2} \frac{v^{\Psi}}{\Psi} \ominus(-1) \int_{0}^{v}\left(\int_{0}^{v} \Phi\left(r, v(r), v^{\Psi}(r)\right) r^{\Psi-1} d r\right) r^{\Psi-1} d r+\eta_{1},
$$

if $v$ is differentiable of type ( $\Psi-2)$ and $v^{\Psi}$ is differentiable of type ( $\left.\Psi-1\right)$.

Proof. We provide the proof of case (1) only. Other cases can be proved similarly.

Since a continuous fuzzy-valued function is integrable, so fuzzy conformable integral of $\Phi$ must exist. Now integrating both sides of the equation (4.1), we have

$$
v^{\Psi}(v)=\int_{0}^{v} \Phi\left(r, v(r), v^{\Psi}(r)\right) r^{\Psi-1} d r+v^{\Psi}(0)
$$

Using the initial condition $v^{\Psi}(0)=\eta_{2}$, we have,

$$
v^{\Psi}(v)=\int_{0}^{v} \Phi\left(r, v(r), v^{\Psi}(r)\right) r^{\Psi-1} d r+\eta_{2} .
$$

Taking integration again, we obtain

$$
\left.v(v)=\int_{0}^{v}\left(\int_{0}^{v} \Phi\left(r, v(r), v^{\Psi}(r)\right) r^{\Psi-1} d r\right)+\eta_{2}\right) r^{\Psi-1} d r+v(0) .
$$


Using the initial condition $v(0)=\eta_{1}$, we obtain our required result in the form

$$
v(v)=\int_{0}^{v}\left(\int_{0}^{v} \Phi\left(r, v(r), v^{\Psi}(r)\right) r^{\Psi-1} d r\right) r^{\Psi-1} d r+\eta_{2} \frac{v^{\Psi}}{\Psi}+\eta_{1} .
$$

Now we present the solution of fuzzy conformable initial value problem of order $2 \Psi$ given by

$$
\begin{aligned}
& \Phi^{2 \Psi}(v)+\alpha \odot \Phi^{\Psi}(v)+\beta \odot \Phi(v)=\eta(v), \\
& \Phi(0)=v, \Phi^{\Psi}(0)=\omega,
\end{aligned}
$$

where $\alpha, \beta>0, v, \omega \in \mathbb{R}_{\Phi}$, and $\eta(v)$ is a continuos fuzzy-valued function.

Theorem 4.2. Let $\Phi(v)$ is a solution of the type ( $\Psi-p)$, we translate the equation (4.2) into a system of conformable differential equations of order $2 \Psi$ which is called a system of type ( $\Psi-p)$ for equation (4.2).

System $(\Psi-1)$

$$
\begin{aligned}
\left(\Phi_{*}\right)^{2 \Psi}(v, \gamma)+\alpha \cdot\left(\Phi_{*}\right)^{\Psi}(v, \gamma)+\beta \cdot \Phi_{*}(v, \gamma) & =\eta_{*}(v, \gamma), \\
\left(\Phi^{*}\right)^{2 \Psi}(\nu, \gamma)+\alpha \cdot\left(\Phi^{*}\right)^{\Psi}(v, \gamma)+\beta \cdot \Phi^{*}(v, \gamma) & =\eta^{*}(\nu, \gamma), \\
\Phi_{*}(0, \gamma) & =\left(v_{*}, \gamma\right), \\
\Phi^{*}(0, \gamma) & =\left(v^{*}, \gamma\right), \\
\left(\Phi_{*}\right)^{\Psi}(0, \gamma) & =\left(\omega_{*}, \gamma\right) \\
\left(\Phi^{*}\right)^{\Psi}(0, \gamma) & =\left(\omega^{*}, \gamma\right) .
\end{aligned}
$$

System $(\Psi-2)$

$$
\begin{aligned}
\left(\Phi^{*}\right)^{2 \Psi}(v, \gamma)+\alpha \cdot\left(\Phi_{*}\right)^{\Psi}(v, \gamma)+\beta \cdot \Phi_{*}(v, \gamma) & =\eta_{*}(v, \gamma), \\
\left(\Phi_{*}\right)^{2 \Psi}(v, \gamma)+\alpha \cdot\left(\Phi^{*}\right)^{\Psi}(v, \gamma)+\beta \cdot \Phi^{*}(v, \gamma) & =\eta^{*}(v, \gamma), \\
\Phi_{*}(0, \gamma) & =\left(v_{*}, \gamma\right), \\
\Phi^{*}(0, \gamma) & =\left(v^{*}, \gamma\right), \\
\left(\Phi_{*}\right)^{\Psi}(0, \gamma) & =\left(\omega_{*}, \gamma\right), \\
\left(\Phi^{*}\right)^{\Psi}(0, \gamma) & =\left(\omega^{*}, \gamma\right) .
\end{aligned}
$$

System ( $\Psi-3)$

$$
\begin{aligned}
\left(\Phi^{*}\right)^{2 \Psi}(v, \gamma)+\alpha \cdot\left(\Phi^{*}\right)^{\Psi}(v, \gamma)+\beta \cdot \Phi_{*}(v, \gamma) & =\eta_{*}(v, \gamma), \\
\left(\Phi_{*}\right)^{2 \Psi}(v, \gamma)+\alpha \cdot\left(\Phi_{*}\right)^{\Psi}(v, \gamma)+\beta \cdot \Phi^{*}(v, \gamma) & =\eta^{*}(v, \gamma), \\
\Phi_{*}(0, \gamma) & =\left(v_{*}, \gamma\right), \\
\Phi^{*}(0, \gamma) & =\left(v^{*}, \gamma\right), \\
\left(\Phi^{*}\right)^{\Psi}(0, \gamma) & =\left(\omega_{*}, \gamma\right), \\
\left(\Phi_{*}\right)^{\Psi}(0, \gamma) & =\left(\omega^{*}, \gamma\right) .
\end{aligned}
$$

System $(\Psi-4)$

$$
\begin{aligned}
\left(\Phi_{*}\right)^{2 \Psi}(\nu, \gamma)+\alpha \cdot\left(\Phi^{*}\right)^{\Psi}(\nu, \gamma)+\beta \cdot \Phi_{*}(v, \gamma) & =\eta_{*}(v, \gamma), \\
\left(\Phi^{*}\right)^{2 \Psi}(v, \gamma)+\alpha \cdot\left(\Phi_{*}\right)^{\Psi}(v, \gamma)+\beta \cdot \Phi^{*}(v, \gamma) & =\eta^{*}(v, \gamma), \\
\Phi_{*}(0, \gamma) & =\left(v_{*}, \gamma\right), \\
\Phi^{*}(0, \gamma) & =\left(v^{*}, \gamma\right), \\
\left(\Phi^{*}\right)^{\Psi}(0, \gamma) & =\left(\omega_{*}, \gamma\right), \\
\left(\Phi_{*}\right)^{\Psi}(0, \gamma) & =\left(\omega^{*}, \gamma\right) .
\end{aligned}
$$


Theorem 4.3. If a fuzzy-valued function $\Phi(v)=\left[\Phi_{*}(v), \Phi^{*}(v)\right]$ has valid $\gamma$-cuts and $\Phi_{*}(v), \Phi^{*}(v)$ solve the associated systems, then $\Phi(v)$ is a solution of type $(\Psi-p)$, where $p \in\{1,4\}$.

Example 4.4. Consider fuzzy conformable initial value problem of order $2 \Psi$

$$
\begin{gathered}
\Phi^{2 \Psi}(v)=\eta, \\
\Phi(0)=v, \Phi^{\Psi}(0)=\omega,
\end{gathered}
$$

where Initial conditions $\eta, v$, and $\omega$ are fuzzy numbers with $\gamma$-cuts

$$
(\eta, \gamma)=[1+\gamma, 3-\gamma],(v, \gamma)=[4+\gamma, 6-\gamma],(\omega, \gamma)=[-3+2 \gamma, 1-2 \gamma]
$$

We have four possibilities relative to the four types of differentiability.

1. Associated to the system ( $\Psi-1)$, we have the solution

$$
\begin{aligned}
& \Phi_{*}(\nu, \gamma)=(1+\gamma) \frac{v^{2 \Psi}}{2 \Psi}+(-3+2 \gamma) \frac{\nu^{\Psi}}{\Psi}+(4+\gamma), \\
& \Phi^{*}(\nu, \gamma)=(3-\gamma) \frac{v^{2 \Psi}}{2 \Psi}+(1-2 \gamma) \frac{\nu^{\Psi}}{\Psi}+(6-\gamma) .
\end{aligned}
$$

2. Associated to the system ( $\Psi-2)$, we have the solution

$$
\begin{aligned}
& \Phi^{*}(\nu, \gamma)=(1+\gamma) \frac{v^{2 \Psi}}{2 \Psi}+(4+\gamma) \frac{v^{\Psi}}{\Psi}+(-3+2 \gamma) \\
& \Phi_{*}(\nu, \gamma)=(3-\gamma) \frac{v^{2 \Psi}}{2 \Psi}+(6-\gamma) \frac{\nu^{\Psi}}{\Psi}+(1-2 \gamma) .
\end{aligned}
$$

3. Associated to the system ( $\Psi-3)$, we have the solution

$$
\begin{aligned}
& \Phi^{*}(\nu, \gamma)=(1+\gamma) \frac{v^{2 \Psi}}{2 \Psi}+(1-2 \gamma) \frac{v^{\Psi}}{\Psi}+(6-\gamma), \\
& \Phi_{*}(\nu, \gamma)=(3-\gamma) \frac{v^{2 \Psi}}{2 \Psi}+(-3+2 \gamma) \frac{\nu^{\Psi}}{\Psi}+(4+\gamma) .
\end{aligned}
$$

4. Associated to the system ( $\Psi-4)$, we have the solution

$$
\begin{aligned}
& \Phi^{*}(\nu, \gamma)=(1+\gamma) \frac{v^{2 \Psi}}{2 \Psi}+(-3+2 \gamma) \frac{v^{\Psi}}{\Psi}+(4+\gamma) \\
& \Phi_{*}(\nu, \gamma)=(3-\gamma) \frac{v^{2 \Psi}}{2 \Psi}+(1-2 \gamma) \frac{v^{\Psi}}{\Psi}+(6-\gamma) .
\end{aligned}
$$

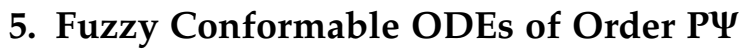

Fuzzy conformable initial value problem of order $p \Psi$ corresponding to the fuzzy conformable differential equation of order $\mathrm{p} \Psi$

$$
\digamma^{p \Psi}(\nu)=\Phi\left(\tau, \nu(\tau), \nu^{\Psi}(\tau), v^{2 \Psi}(\tau), \cdots, v^{(p-1) \Psi}(\tau)\right)
$$

is defined as

$$
\begin{gathered}
\digamma^{{ }^{\Psi \Psi}}(v)=\Phi\left(\tau, v(\tau), v^{\Psi}(\tau), v^{2 \Psi}(\tau), \cdots, v^{(p-1) \Psi}(\tau)\right) \\
v\left(\tau_{0}\right)=\alpha_{1}, v^{\Psi}\left(\tau_{0}\right)=\alpha_{2}, \cdots, v^{(p-1) \Psi}\left(\tau_{0}\right)=\alpha_{p-1} .
\end{gathered}
$$


Theorem 5.1. Fuzzy conformable initial value problem equation (5.1) has a unique solution if for a continuous fuzzy-valued function $\Phi:[0, \infty) \times \mathbb{R}_{\Phi} \times \mathbb{R}_{\Phi} \times \cdots \rightarrow \mathbb{R}_{\Phi},(\exists)$ positive constants $\alpha_{i}$ for $i=1,2, \cdots, p$ such that

$$
\left.\mathrm{D}_{\mathrm{H}}\left(\Phi\left(\tau, v_{1}, v_{2}, \cdots, v_{p}\right)\right), \Phi\left(\tau, \digamma_{1}, \digamma_{2}, \cdots, \digamma_{p}\right)\right) \leqslant \sum \alpha_{i} D_{H}\left(v_{i}, \digamma_{i}\right),
$$

where $v_{i}$ and $\digamma_{i}$ are fuzzy numbers.

Theorem 5.2. For a continuous fuzzy-valued function $\Phi:[0, \infty) \times \mathbb{R}_{\Phi} \times \mathbb{R}_{\Phi} \times \cdots \rightarrow \mathbb{R}_{\Phi}, a$ fuzzy-valued function $F$ is a solution of equation (5.1) iff following equation is satisfied

$$
\begin{aligned}
\digamma(\tau)= & \alpha_{0}+\alpha_{1} \frac{\beta_{1}\left(\tau-\tau_{0}\right)^{\Psi}}{\Psi}+\alpha_{2} \frac{\beta_{2}\left(\tau-\tau_{0}\right)^{2 \Psi}}{2 \Psi !} \\
& +\cdots+\alpha_{(p-1)} \frac{\beta_{(p-1)}}{(p-1) \Psi !}\left(\tau-\tau_{0}\right)(p-1) ! \Psi \\
& +\alpha_{p} \int_{\tau_{0}}^{\tau} \int_{\tau_{0}}^{\tau} \int_{\tau_{0}}^{\tau} \Phi\left(\tau, v(\tau), \nu^{\Psi}(\tau), \ldots, v^{(p-1) \Psi}(\tau)\right) \tau^{(\Psi-1) p !} d \tau, \cdots, d \tau,
\end{aligned}
$$

where $\alpha_{i}=\left\{\begin{array}{l}1 \text { if } \digamma^{i \Psi}(\eta) \text { is differentiable of type }(\Psi-1), \\ \ominus(-1) \text { if } \digamma^{i \Psi}(\eta) \text { is differentiable of type }(\Psi-2) .\end{array}\right.$

Proof. Since $\Phi$ is fuzzy conformable continuous, so fuzzy conformable integral of $\Phi$ must exist. The solution of the fuzzy conformable differential equation of order $p \Psi$

$$
\digamma^{(p+1) \Psi}(v)=\Phi\left(\tau, v(\tau), v^{\Psi}(\tau), v^{2 \Psi}(\tau), \cdots, v^{p \Psi}(\tau)\right),
$$

has the solution of the form

$$
\begin{array}{r}
\digamma^{\Psi}(\tau)=\alpha_{1}+\alpha_{2} \frac{\beta_{2}\left(\tau-\tau_{0}\right)^{2 \Psi}}{2 \Psi !}+\cdots+\alpha_{(p-2)} \frac{\beta_{(p-2)}\left(\tau-\tau_{0}\right)^{(p-2) \Psi}}{(p-2) \Psi !}+ \\
\left.\alpha_{(p-1)} \int_{\tau_{0}}^{\tau} \ldots \int_{\tau_{0}}^{\tau} \Phi\left(r, v(r), \nu^{\Psi}(r), \cdots, \nu^{p \Psi}(r)\right) d r, \cdots, d_{r r}{ }^{p} \Psi-1\right)
\end{array}
$$

Now integrating both sides of the above expression, we obtain our required result.

Example 5.3. Consider fuzzy conformable initial value problem

$$
\begin{aligned}
& \Phi^{2 \Psi}(v)=\Phi(v), \\
& \Phi(0)=v, \Phi^{\Psi}(0)=\omega,
\end{aligned}
$$

where Initial conditions $v$, and $\omega$ are fuzzy numbers with $\gamma$-cuts have the form

$$
(v, \gamma)=[\gamma-1,1-\gamma],(\omega, \gamma)=[\gamma, 2-\gamma]
$$

Now, integrating on both sides of the equation (5.3), we have

$$
\Phi(v, \gamma)=[\gamma-1,1-\gamma]+\alpha_{2}[\gamma, 2-\gamma] \cdot \frac{\nu^{\Psi}}{\Psi}+\alpha_{1} \int_{0}^{v} \int_{0}^{\nu} \Phi(\mathrm{q}) \mathrm{q}^{2(\Psi-1)} \mathrm{dqdq} .
$$

Now, we have four possibilities relative to the four types of differentiability. 


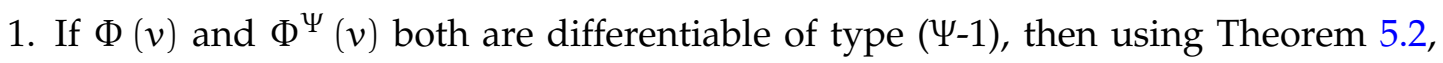
we have

$$
\Phi(\nu, \gamma)=[\gamma-1,1-\gamma]+[\gamma, 2-\gamma] \cdot \frac{\nu^{\Psi}}{\Psi}+\int_{0}^{\nu} \int_{0}^{\nu} \Phi(\mathrm{q}) \mathrm{q}^{2(\Psi-1)} \mathrm{dqdq}
$$

Now, solving the above equation, we have the solution in the form

$$
\Phi(\nu, \gamma)=\left[(\gamma-1)+\gamma \cdot \frac{\nu^{\Psi}}{\Psi}+(\gamma-1) \cdot \frac{v^{2 \Psi}}{2 \Psi},(1-\gamma)+(2-\gamma) \cdot \frac{\nu^{\Psi}}{\Psi}+(1-\gamma) \cdot \frac{v^{2 \Psi}}{2 \Psi}\right]
$$

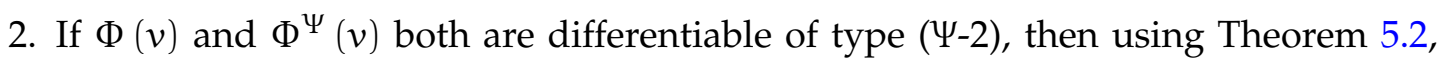
we have

$$
\Phi(\nu, \gamma)=[\gamma-1,1-\gamma] \ominus(-1)[\gamma, 2-\gamma] \cdot \frac{\nu^{\Psi}}{\Psi}+\int_{0}^{\nu} \int_{0}^{\nu} \Phi(\mathrm{q}) \mathrm{q}^{2(\Psi-1)} \mathrm{dqdq} .
$$

Now, solving the above equation, we have the solution in the form

$$
\Phi(\nu, \gamma)=\left[(\gamma-1)+(2-\gamma) \cdot \frac{\nu^{\Psi}}{\Psi}+(\gamma-1) \cdot \frac{v^{2 \Psi}}{2 \Psi},(1-\gamma)+\gamma \cdot \frac{\nu^{\Psi}}{\Psi}+(1-\gamma) \cdot \frac{v^{2 \Psi}}{2 \Psi}\right]
$$

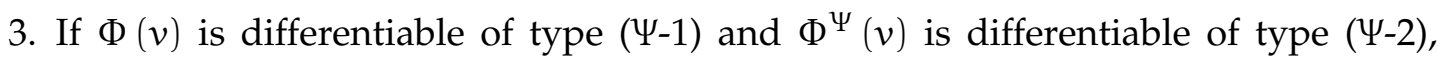
then using Theorem 5.2, we have

$$
\Phi(v, \gamma)=[\gamma-1,1-\gamma] \ominus(-1)[\gamma, 2-\gamma] \cdot \frac{\nu^{\Psi}}{\Psi} \ominus(-1) \int_{0}^{\nu} \int_{0}^{\nu} \Phi(\mathbf{q}) \mathrm{q}^{2(\Psi-1)} \mathrm{dqd} \mathbf{q} .
$$

Now, solving the above equation, we have the solution in the form

$$
\Phi(v, \gamma)=\left[(\gamma-1)+(2-\gamma) \cdot \frac{v^{\Psi}}{\Psi}+(1-\gamma) \cdot \frac{v^{2 \Psi}}{2 \Psi},(1-\gamma)+\gamma \cdot \frac{v^{\Psi}}{\Psi}+(\gamma-1) \cdot \frac{v^{2 \Psi}}{2 \Psi}\right]
$$

4. If $\Phi(v)$ is differentiable of type $(\Psi-2)$ and $\Phi^{\Psi}(\nu)$ is differentiable of type ( $\left.\Psi-1\right)$, then using Theorem 5.2, we have

$$
\Phi(\nu, \gamma)=[\gamma-1,1-\gamma]+[\gamma, 2-\gamma] \cdot \frac{\nu^{\Psi}}{\Psi} \ominus(-1) \int_{0}^{\nu} \int_{0}^{\nu} \Phi(\mathbf{q}) \mathrm{q}^{2(\Psi-1)} \mathrm{dqdq} .
$$

Now, solving the above equation, we have the solution in the form

$$
\Phi(\nu, \gamma)=\left[(\gamma-1)+\gamma \cdot \frac{\nu^{\Psi}}{\Psi}+(1-\gamma) \cdot \frac{v^{2 \Psi}}{2 \Psi},(1-\gamma)+(2-\gamma) \cdot \frac{\nu^{\Psi}}{\Psi}+(\gamma-1) \cdot \frac{v^{2 \Psi}}{2 \Psi}\right]
$$

\section{Conclusion and summary}

We have used a newly defined conformable strongly generalized type derivative to solve fuzzy conformable ordinary differential equations of order $\Psi, 2 \Psi$ and then also generalized our results to solve fuzzy conformable differential equations of order $p \Psi$. Also, we have defined relevant results on fuzzy conformable calculus which are needed 
to solve fuzzy conformable ordinary differential equations. It seems to be the first approach to solve fuzzy conformable ordinary differential equations with an analytical method. So we obtain the solution that has a decreasing length of support. Our future goal is to define the fuzzy conformable partial derivative and then use it to solve the fuzzy conformable partial differential equations.

Acknowledgment. The author T. Abdeljawad would like to thank Prince Sultan University for the moral support through the research group Nonlinear Analysis Methods in Applied Mathematics (NAMAM) group number RG-DES-2017-01-17.

\section{References}

[1] Byatt WJ, Kandel A (1978). Fuzzy differential equations. In Proceedings of the International Conference on Cybernetics and Society, Tokyo, Japan.

[2] Afzal A, Ansari Z, Alshahrani S, Raj AK, Kuruniyan MS, Saleel CA, Nisar KS (2021). Clustering of COVID-19 data for knowledge discovery using c-means and fuzzy c-means. Results in Physics, 29: 104639. https://doi.org/10.1016/j.rinp.2021.104639

[3] Puri ML, Ralescu DA (1983). Differentials of fuzzy functions. Journal of Mathematical Analysis and Applications, 91(2): 552-558. https ://doi .org/10.1016/0022-247X (83) 90169-5

[4] Kaleva O (1987). Fuzzy differential equations. Fuzzy sets and systems, 24(3): 301-317. https ://doi .org/ 10.1016/0165-0114(87) 90029-7

[5] Seikkala S (1987). On the fuzzy initial value problem. Fuzzy sets and systems, 24(3): 319-330. https: //doi.org/10.1016/0165-0114(87)90030-3

[6] Bede B (2008). Note on Numerical solutions of fuzzy differential equations by predictor-corrector method. Information sciences, 178(7): 1917-1922. https://doi.org/10.1016/j.ins. 2007.11.016

[7] Pirzada UM (2018). Generalized Seikkala Differentiability and its Application to Fuzzy initial value problem. arXiv preprint arXiv:1812.04963.

[8] Devi SS, Ganesan K (2019). Application of linear fuzzy differential equation in day to day life. AIP Conference Proceedings, 2112(1): 020169. https://doi.org/10.1063/1.5112354

[9] Vasavi Ch, Kumar GS, Rao TS, Rao BV (2017). Application of fuzzy differential equations for cooling problems. International Journal of Mechanical Engineering and Technology, 8(2): 712-721.

[10] Kumar A, Malik M, Nisar KS (2021). Existence and total controllability results of fuzzy delay differential equation with non-instantaneous impulses. Alexandria Engineering Journal, 60(6): 6001-6012. http://dx. doi.org/10.1016/j.aej.2021.04.017

[11] Rashid S, Akdemir AO, Nisar KS, Abdeljawad K, Rahman G (2020). New generalized reverse Minkowski and related integral inequalities involving generalized fractional conformable integrals. Journal of Inequalities and Applications, 2020(1): 1-15. https://doi.org/10.1186/s13660-020-02445-2

[12] Al-Mdallal QM, Hajji MA, Abdeljawad T (2021). On the iterative methods for solving fractional initial value problems: new perspective. Journal of Fractional Calculus and Nonlinear Systems, 2(1): 76-81. https://doi.org/10.48185/jfcns.v2i1.297

[13] Khalil R, Al Horani M, Yousef A, Sababheh M (2014). A new definition of fractional derivative. Journal of Computational and Applied Mathematics, 264: 65-70. https ://doi .org/10.1016/j.cam. 2014.01. 002

[14] Khalil R, Al Horani M, Hammad MA (2019). Geometric meaning of conformable derivative via fractional cords. Journal of Mathematics and Computer Science, 19: 241-245. http://dx.doi.org/10.22436/ jmcs.019.04.03

[15] Abdelhakim AA, Machado JAT (2019). A critical analysis of the conformable derivative. Nonlinear Dynamics, 95(4): 3063-3073. https : //doi .org/10.1007/s11071-018-04741-5

[16] Bohner M, Hatip VF (2018). Dynamic cobweb models with conformable fractional derivatives. Mathematical Methods in the Applied Sciences, 41(18): 9010-9017. https://doi.org/10.1016/j.nahs.2018.09.004

[17] $\mathrm{Ma} \mathrm{X}, \mathrm{Wu} \mathrm{W}$, Zeng B, Wang Y, Wu X (2020). The conformable fractional grey system model. ISA transactions, 96: 255-271. https://doi.org/10.1016/j.isatra.2019.07.009

[18] Martinez L, Rosales JJ, Carreno CA, Lozano JM (2018). Electrical circuits described by fractional conformable derivative. International Journal of Circuit Theory and Applications, 46(5): 1091-1100. https: //doi.org/10.1002/cta.2475 
[19] Younus A, Abdeljwad T, Gul T (2020). On Stability Criteria of Fractal Differential Systems of Conformable Type. Fractals 28(8). http://dx. doi.org/10.1142/S0218348X20400095

[20] Younas A, Abdeljawad T, Batool R, Zehra A, Alqudah AL (2020). Linear conformable differential system and its controllability. Advances in Difference Equations 2020(1): 1-26. https://dx.doi.org/10.1186/ s13662-020-02899-0

[21] Younus A, Bukhsh K, Tunç C (2020). Existence of resolvent for conformable fractional Volterra integral equations. Applications and Applied Mathematics, 15(1): 372-393.

[22] Nisar KS, Ilhan OA, Manafian J, Shahriari M, Soybas D (2021). Analytical behavior of the fractional Bogoyavlenskii equations with conformable derivative using two distinct reliable methods. Results in Physics, 22(3): 103975. https://doi.org/10.1016/j.rinp.2021.103975

[23] Napoles JEN, Guzmán PM, Bittencurt LMLM (2020). A note on the qualitative behavior of some nonlinear local improper conformable differential equations. Journal of Fractional Calculus and Nonlinear Systems, 1(1): 13-20. http://dx.doi.org/10.48185/jfcns.v1i1.48

[24] Arqub OA, Al-Smadi M (2020). fuzzy conformable fractional differential equations: Novel extended approach and new numerical solutions. Soft Computing, 2020: 1-22. https://doi.org/10.1007/ s00500-020-04687-0

[25] Harir A, Melliani S, Chadli LS (2020). Fuzzy Generalized Conformable Fractional Derivative. Advances in Fuzzy Systems, 2020(4): 1-7. https://doi.org/10.1155/2020/1954975

[26] Al-Smadi MH (2020). Numerical Simulation of Conformable Fuzzy Differential Equations. In The International Conference on Mathematical and Related Sciences: 108-122. http://dx.doi.org/10.1007/ 978-3-030-43002-3_10

[27] Armand A, Allahviranloo T, Gouyandeh Z (2018). Some fundamental results on fuzzy calculus. Iranian Journal of Fuzzy Systems, 15(3): 27-46. https : //doi .org/10.22111/IJFS . 2018. 3948

[28] Bede B, Rudas IJ, Bencsik AL (2007). First order linear fuzzy differential equations under generalized differentiability. Information sciences, 17(7): 1648-1662. https://doi.org/10.1016/j.ins. 2006.08.021

[29] Chalco-Cano Y, Roman-Flores H (2008). On new solutions of fuzzy differential equations. Chaos, Solitons Fractals, 38(1): 112-119. https://doi .org/10.1016/j . chaos . 2006.10.043

[30] Chang Sheldon SL, Zadeh LA (1996). On fuzzy mapping, and control. In Fuzzy sets, fuzzy logic, and fuzzy systems: 180-184. https://doi.org/10.1142/9789814261302_0012

[31] Eltayeb H, Mesloub S, Abdalla YT, Kilicman A (2019). A Note on Double conformable Laplace transform Method and Singular One Dimensional conformable Pseudohyperbolic Equations. Mathematics, 7(10): 949. https://doi.org/10.3390/math7100949

[32] Georgiou DN, Kougias IE (2004). On Cauchy problems for fuzzy differential equations. International Journal of Mathematics and Mathematical Sciences, 2004(15): 799-805. https://doi.org/10.1155/ S0161171204202277

[33] Hooshangian L, Allahviranloo T (2014). A new method to find fuzzy N th order derivation and applications to fuzzy $\mathrm{N}$ th order arithmetic based on generalized $\mathrm{H}$-derivation. An International Journal of Optimization and Control Theories Applications, 4(2): 105-121. https://doi.org/10.11121/ijocta.01.2014.00183

[34] Khastan A, Rodríguez-López R (2016). On the solutions to first order linear fuzzy differential equations. fuzzy Sets and Systems, 295: 114-135. https://doi.org/10.1016/j.fss.2015.06.005

[35] Khastan A, Bahrami F, Ivaz K (2009). New results on multiple solutions for Nth-order fuzzy differential equations under generalized differentiability. Boundary value problems, 2009: 1-13. https://doi .org/10. $1155 / 2009 / 395714$

[36] Salahshour S, Allahviranloo T (2013). Applications of fuzzy Laplace transforms. Soft computing, 17(1): 145-158. https://doi.org/10.1007/s00500-012-0907-4

[37] Salgado S, Barros LCD, Esmi E, Eduardo DE (2019). Solution of a fuzzy differential equation with interactivity via Laplace transform. Journal of Intelligent and Fuzzy Systems, 37(2): 2495-2501. http: //dx.doi.org/10.3233/JIFS-182761 